\title{
Chebyshev Wavelet Method for Numerical Solutions of Coupled Burgers' Equation
}

\author{
Ö. Oruç*1, F. Bulut ${ }^{2}$, A. Esen ${ }^{3}$ \\ ${ }^{1}$ Eğil Vocational and Technical Anatolian High School, Diyarbakır, Turkey \\ ${ }^{2}$ Inonu University, Department of Physics, Malatya, Turkey \\ ${ }^{3}$ Inonu University, Department of Mathematics, Malatya, Turkey
}

\begin{abstract}
This paper deals with the numerical solutions of one dimensional time dependent coupled Burgers' equation with suitable initial and boundary conditions by using Chebyshev wavelets in collaboration with a collocation method. The proposed method converts coupled Burgers' equations into system of algebraic equations by aid of the Chebyshev wavelets and their integrals which can be solved easily with a solver. Benchmarking of the proposed method with exact solution and other known methods already exist in the literature is made by three test problems. The feasibility of the proposed method is demonstrated by test problems and indicates that the proposed method gives accurate results in short cpu times. Computer simulations show that the proposed method is computationally cheap, fast and quite good even in the case of less number of collocation points.
\end{abstract}

Mathematics Subject Classification (2010). 35Q35, 65M70

Keywords. Chebyshev wavelet method, Chebyshev collocation, coupled Burgers' equation, nonlinear phenomena, numerical solution

\section{Introduction}

We consider the Coupled Burgers' problem

$$
\begin{aligned}
u_{t}-u_{x x}+\eta u u_{x}+\alpha(u . v)_{x}=0, & & x \in[0,1], & t \in[0, T] \\
v_{t}-v_{x x}+\xi v v_{x}+\beta(u . v)_{x}=0, & & x \in[0,1], & t \in[0, T]
\end{aligned}
$$

with the initial conditions

$$
u(x, 0)=\psi_{1}(x), \quad v(x, 0)=\psi_{2}(x), \quad x \in[a, b]
$$

and the boundary conditions

$$
\begin{array}{lll}
u(0, t)=f_{1}(t), & u(1, t)=f_{2}(t), & t \in[0, T] \\
v(0, t)=g_{1}(t), & v(1, t)=g_{2}(t), & t \in[0, T]
\end{array}
$$

\footnotetext{
*Corresponding Author.

Email addresses: omeroruc0@gmail.com (Ö. Oruç), fatih.bulut@inonu.edu.tr (F. Bulut), alaattin.esen@inonu.edu.tr (A. Esen)

Received: 16.02.2017; Accepted: 12.07.2017
} 
where $\eta, \xi$ are real constants and $\alpha, \beta$ are arbitrary constants depend on system parameters such as Peclet number, Stokes velocity of particles due to gravity and the Brownian diffusivity [30]. $u(x, t)$ and $v(x, t)$ are the velocity components to be determined; $\psi_{i}, f_{i}$ and $g_{i}(i=1,2)$ are the known functions; $u u_{x}$ is the nonlinear convection term, $u_{t}$ is unsteady term and $u_{x x}$ is diffusion term.

Coupled Burgers' equation was first derived by Esipov [12] which is a simple model of sedimentation or evolution of scaled volume concentrations of two kinds of particles in fluid suspensions or colloids, under the effect of gravity [30]. This equation has been solved by various approaches such as; Khater et al. [21] used the Chebyshev spectral collocation method to solve the equation and M. Dehghan et al. [11] applied Adomian-Pade technique for solving the coupled Burgers equations and more recently Kutluay and Ucar [23] solved coupled Burgers' equation by using the Galerkin quadratic B-spline method. In order to solve Eqs. (1.1), (1.2) Mittal and Arora [28] used a cubic B-spline collocation scheme. Rashid and Ismail [34] have used Fourier Pseudospectral method to solve the equation numerically. Srivastava et al. [42] obtained numerical solutions of the Eqs.(1.1), (1.2) by implicit finite-difference method. Zhang et al. [37] applied local discontinuous Galerkin method to solve coupled Burgers' equations. Siraj-ul-Islam et al. [17] solved coupled Burgers' equation by mesh free interpolation method. Kelleci and Ylldırım [20] have solved the equation by combining homotopy perturbation and Pade techniques and Inan et al. [16] have applied Bäcklund transformation to the Eqs.(1.1), (1.2). In the studies [22,29], coupled Burgers' equations are solved by Haar wavelet method. Rashid et al. have solved the coupled viscous Burgers' equation by Chebyshev-Legendre Pseudo-Spectral method in [33].

Kaya [19] obtained the exact solution of the equation by Adomian Decomposition method and Soliman [41] used a modified extended tanh-function method to obtain its exact solution. Abdou and Soliman [2] used Variational iteration method to solve the coupled viscous Burgers' equation.

The wavelet methods were first applied for solving differential equations at the beginning of 1990s. Until now a vast number of papers devoted to this topic. In most cases the wavelet coefficients were calculated by the Galerkin or collocation method. But there is a drawback in these methods since we have to evaluate integrals of some combinations of the wavelet functions (connection coefficients). This is a very sophisticated problem, since for most wavelet families we do not have an explicit form for these integrals [25]. Due to these facts, researchers have focused on more simple wavelets such as Haar wavelets, Legendre wavelets and Chebyshev wavelets for obtaining numerical solutions of differential and integral equations. There are a lot of studies on application of Haar wavelets in solving differential and integral equations numerically $[6,7,9,18,22,24,26,27,29,31,32,40]$. Nowadays, Legendre and Chebyshev wavelets are studied by many researchers $[3,4,8,13$, $14,35,36,38,39,44-47]$. In this paper we propose a Chebyhev wavelet method for solving coupled Burgers' equations numerically.

The outline of this paper is as follows. In Section 2, preliminaries about Chebyshev wavelets are given. In section 3, we show how to use Chebyshev wavelet method for solving coupled Burgers' equation. In Section 4, proposed method tested by three examples, obtained numerical results tabulated and numerical solutions depicted graphically. Finally we conclude the paper in Section 5.

\section{Preliminaries and notations}

In this section, we give some necessary definitions and mathematical preliminaries of Chebyshev wavelets. 


\subsection{Chebyshev wavelets}

Wavelets constitute a family of functions which are generated from dilation and translation of a single function which is called as mother wavelet $\psi(x)$. If the dilation parameter $a$ and the translation parameter $b$ vary continuously we have the following family of continuous wavelets [10]:

$$
\psi_{a, b}(x)=|a|^{-1 / 2} \psi\left(\frac{x-b}{a}\right),
$$

where $a, b \in \mathbb{R}$ and $a \neq 0$. Chebyshev wavelets $\psi_{n m}=\psi(k, n, m, x)$ defined as follows:

$$
\psi_{n m}(x)= \begin{cases}\gamma_{m} \frac{2^{(k-1) / 2}}{\sqrt{\pi}} T_{m}\left(2^{k} x-2 n+1\right), & \frac{n-1}{2^{k-1}} \leq x<\frac{n}{2^{k-1}} \\ 0, & \text { else }\end{cases}
$$

where

$$
\gamma_{m}= \begin{cases}\sqrt{2}, & m=0 \\ 2, & m=1,2, \ldots\end{cases}
$$

and $m=0,1, \ldots, M-1$. Here $n=1,2, \ldots, 2^{k-1}, k$ can take any positive integer, $m$ is the degree of Chebyshev polynomials of first kind and $x$ is the normalized time. $T_{m}(x)$ are Chebyshev polynomials of the first kind of degree $m$ and satisfy the following recursive formula:

$$
T_{0}(x)=1, T_{1}(x)=x, \quad T_{m+1}(x)=2 x T_{m}(x)-T_{m-1}(x) .
$$

which are orthogonal with respect to the weight function $\omega(x)=1 / \sqrt{1-x^{2}}$. We should remind that Chebyshev wavelets are orthogonal with respect to the weight function $\omega_{n}(x)=$ $\omega\left(2^{k} x-2 n+1\right)$.

\subsection{Function approximation}

Any function $u(x) \in L_{\omega}^{2}[0,1)$ can be expanded into Chebyshev wavelets as follows:

$$
u(x)=\sum_{n=1}^{\infty} \sum_{m=0}^{\infty} c_{n m} \psi_{n m}(x) .
$$

Here wavelet coefficients are $c_{n m}=\left\langle u(x), \psi_{n m}(x)\right\rangle$, where $\langle.,$.$\rangle represents the inner prod-$ uct with respect to $\omega_{n}(x)$.

In practice, one needs the truncated version of the Eq. (2.2), namely:

$$
u(x)=\sum_{n=1}^{2^{k-1}} \sum_{m=0}^{M-1} c_{n m} \psi_{n m}(x)=C^{T} \Psi(x),
$$

where $C$ and $\Psi(x)$ are $2^{k-1} M \times 1$ matrices given as

$$
\begin{gathered}
C=\left[c_{10}, c_{11}, \ldots, c_{1(M-1)}, c_{20}, c_{21}, \ldots, c_{2(M-1)}, \ldots,\right. \\
\left.c_{2^{k-1} 0}, c_{2^{k-1} 1}, \ldots, c_{2^{k-1}(M-1)}\right]^{T}, \\
\Psi(x)=\left[\psi_{10}(x), \psi_{11}(x), \ldots, \psi(x)_{1(M-1)}, \psi_{20}(x), \psi_{21}(x), \ldots, \psi(x)_{2(M-1)}, \ldots,\right. \\
\left.\psi_{2^{k-1} 0}(x), \psi_{2^{k-1} 1}(x), \ldots, \psi(x)_{2^{k-1}(M-1)}\right] .
\end{gathered}
$$

Convergence analysis of Chebyshev wavelets is given in $[3,45]$. 


\subsection{Integrals of Chebyshev wavelets [8]}

We denote the first integral of the Eq. (2.1) as $p_{n m}(x)=\int_{0}^{x} \psi_{n m}(s) d s$ and the second integral of the Eq. (2.1) as $q_{n m}(x)=\int_{0}^{x} p_{n m}(s) d s$. The first integral $p_{n m}(x)$ is given for $m=0, m=1$ and $m>1$ as follows:

$$
\begin{gathered}
p_{n 0}(x)= \begin{cases}0 & 0 \leq x<\frac{n-1}{2^{k-1}} \\
\gamma_{0} \frac{2^{-(k-1) / 2-1}}{\sqrt{\pi}}\left[T_{1}(t)+T_{0}(t)\right], & \frac{n-1}{2^{k-1} \leq x<\frac{n}{2^{k-1}}} \\
\gamma_{0} \frac{2^{-(k-1) / 2}}{\sqrt{\pi}} T_{0}(t), & \frac{n}{2^{k-1} \leq x<1}\end{cases} \\
p_{n 1}(x)= \begin{cases}0 & 0 \leq x<\frac{n-1}{2^{k-1}} \\
\gamma_{1} \frac{2^{-(k-1) / 2-3}}{\sqrt{\pi}}\left[T_{2}(t)-T_{0}(t)\right], & \frac{n-1}{2^{k-1} \leq x<\frac{n}{2^{k-1}}} \\
0, & \frac{n}{2^{k-1} \leq x<1}\end{cases} \\
p_{n m}(x)= \begin{cases}0 \quad \\
\gamma_{m} \frac{2^{-(k-1) / 2-2}}{\sqrt{\pi}}\left[\frac{T_{m+1}(t)-(-1)^{m+1}}{m+1}-\frac{T_{m-1}(t)-(-1)^{m-1}}{m-1}\right], & \frac{n-1}{2^{k-1}} \leq x<\frac{n}{2^{k-1}} \\
\gamma_{m} \frac{2^{-(k-1) / 2-2}}{\sqrt{\pi}}, & \frac{n}{2^{k-1}} \leq x<1\end{cases}
\end{gathered}
$$

where $t=2^{k} x-2 n+1$. The second integral $q_{n m}(x)$ is given for $m=0, m=1, m=2$ and $m>2$ as follows:

$$
\begin{gathered}
q_{n 0}(x)= \begin{cases}0 & 0 \leq x<\frac{n-1}{2^{k-1}} \\
\gamma_{0} \frac{2^{-3(k-1) / 2-4}}{\sqrt{\pi}}\left[T_{2}(t)+4 T_{1}(t)+3 T_{0}(t)\right], & \frac{n-1}{2^{k-1} \leq x<\frac{n}{2^{k-1}}} \\
\gamma_{0} \frac{2^{-(k-1) / 2}}{\sqrt{\pi}}\left(\frac{1}{2^{k}}+x-\frac{n}{2^{k-1}}\right), & \frac{n}{2^{k-1} \leq x<1}\end{cases} \\
q_{n 1}(x)= \begin{cases}0 & 0 \leq x<\frac{n-1}{2^{k-1}} \\
\gamma_{1} \frac{2^{-3(k-1) / 2-4}}{\sqrt{\pi}}\left[\frac{T_{3}(t)}{6}-\frac{3 T_{1}(t)}{2}-\frac{4 T_{0}(t)}{3}\right], & \frac{n-1}{2^{k-1} \leq x<\frac{n}{2^{k-1}}} \\
\gamma_{1} \frac{2^{-3(k-1) / 2-1}}{-3 \sqrt{\pi}}, & \frac{n}{2^{k-1}} \leq x<1\end{cases} \\
q_{n 2}(x)= \begin{cases}0 & 0 \leq x<\frac{n-1}{2^{k-1}} \\
\gamma_{2} \frac{2^{-3(k-1) / 2-3}}{\sqrt{\pi}}\left[\frac{T_{4}(t)-1}{24}-\frac{T_{2}(t)-1}{3}-\frac{2 T_{1}(t)}{3}-\frac{2 T_{0}(t)}{3}\right], & \frac{n-1}{2^{k-1}} \leq x<\frac{n}{2^{k-1}} \\
\gamma_{2} \frac{2^{-(k-1) / 2}}{-3 \sqrt{\pi}}\left(\frac{1}{2^{k}}+x-\frac{n}{2^{k-1}}\right), & \frac{n}{2^{k-1}} \leq x<1\end{cases}
\end{gathered}
$$




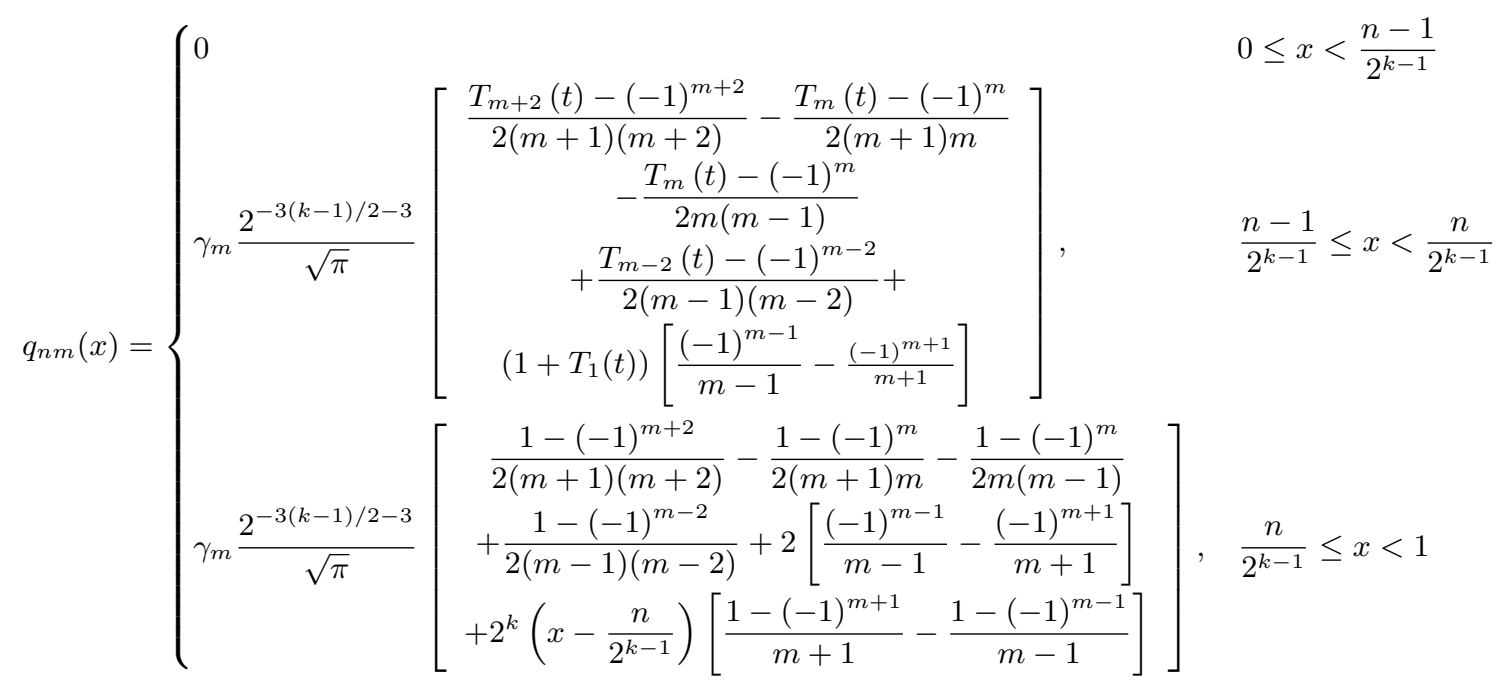

where $t=2^{k} x-2 n+1$. We will use these integrals in solution procedure later.

\section{Method of solution for coupled Burgers' equation}

Consider the equations (1.1), (1.2) with the initial conditions

$$
u(x, 0)=\psi_{1}(x), \quad v(x, 0)=\psi_{2}(x), \quad x \in[0,1]
$$

and the boundary conditions

$$
\begin{array}{lll}
u(0, t)=f_{1}(t), & u(1, t)=f_{2}(t), & t \in[0, T] \\
v(0, t)=g_{1}(t), & v(1, t)=g_{2}(t), & t \in[0, T]
\end{array}
$$

Let us divide the interval $[0, T]$ into $N$ equal parts of length $\Delta t=T / N$ and denote $t_{s}=(s-1) \Delta t, s=1,2, \ldots, N$. In order to use the Chebyshev integrals given in the previous section we expand the highest derivatives that appeared in the Eqs. (1.1) and (1.2) into Chebyshev wavelets. Therefore assume that $\dot{u}^{\prime \prime}(x, t)$ and $\dot{v}^{\prime \prime}(x, t)$ can be expanded in terms of Chebyshev wavelets as

$$
\begin{aligned}
& \dot{u}^{\prime \prime}(x, t)=\sum_{n=1}^{2^{k-1}} \sum_{m=0}^{M-1} c_{n m} \psi_{n m}(x) \\
& \dot{v}^{\prime \prime}(x, t)=\sum_{n=1}^{2^{k-1}} \sum_{m=0}^{M-1} d_{n m} \psi_{n m}(x)
\end{aligned}
$$

where . and ' means differentiation with respect to $t$ and $x$, respectively, the row vectors $c_{n m}$ and $d_{n m}$ are constants in the sub-interval $t \in\left[t_{s}, t_{s+1}\right]$. We discretize $u(x, t)$ below, same procedure can be applied to $v(x, t)$. 
Integrating equation (3.1) with respect to $t$ from $t_{s}$ to $t$ and twice with respect to $x$ from 0 to $x$, we have following equations:

$$
\begin{aligned}
u^{\prime \prime}(x, t)= & \left(t-t_{s}\right) \sum_{n=1}^{2^{k-1}} \sum_{m=0}^{M-1} c_{n m} \psi_{n m}(x)+u^{\prime \prime}\left(x, t_{s}\right), \\
u^{\prime}(x, t)= & \left(t-t_{s}\right) \sum_{n=1}^{2^{k-1}} \sum_{m=0}^{M-1} c_{n m} p_{n m}(x)+u^{\prime}\left(x, t_{s}\right)-u^{\prime}\left(0, t_{s}\right)+u^{\prime}(0, t), \\
u(x, t)= & \left(t-t_{s}\right) \sum_{n=1}^{2^{k-1}} \sum_{m=0}^{M-1} c_{n m} q_{n m}(x)+u\left(x, t_{s}\right)-u\left(0, t_{s}\right) \\
& +x\left[u^{\prime}(0, t)-u^{\prime}\left(0, t_{s}\right)\right]+u(0, t), \\
\dot{u}(x, t)= & \sum_{n=1}^{2^{k-1}} \sum_{m=0}^{M-1} c_{n m} q_{n m}(x)+\dot{u}(0, t)+x \dot{u}^{\prime}(0, t) .
\end{aligned}
$$

By using boundary conditions, we obtain

$$
\begin{array}{lll}
u(0, t)=f_{1}(t), & u\left(0, t_{s}\right)=f_{1}\left(t_{s}\right), & \dot{u}\left(0, t_{s}\right)=f_{1}^{\prime}\left(t_{s}\right) \\
u(1, t)=f_{2}(t), & u\left(1, t_{s}\right)=f_{2}\left(t_{s}\right), & \dot{u}\left(1, t_{s}\right)=f_{2}^{\prime}\left(t_{s}\right)
\end{array}
$$

At $x=1$ in the formulae (3.5) and (3.6) and by using conditions, we have

$$
\begin{aligned}
u^{\prime}(0, t)-u^{\prime}\left(0, t_{s}\right)= & -\left(t-t_{s}\right) \sum_{n=1}^{2^{k-1}} \sum_{m=0}^{M-1} c_{n m} q_{n m}(1)+f_{2}(t) \\
& -f_{2}\left(t_{s}\right)+f_{1}\left(t_{s}\right)-f_{1}(t) \\
\dot{u}^{\prime}(0, t)= & -\sum_{n=1}^{2^{k-1}} \sum_{m=0}^{M-1} c_{n m} q_{n m}(1)-f_{1}^{\prime}(t)+f_{2}^{\prime}(t) .
\end{aligned}
$$

Substituting (3.7) and (3.8) into (3.4)-(3.6) and discretizing the results by assuming $x \rightarrow x_{l}$ and $t \rightarrow t_{s+1}$ we obtain

$$
\begin{aligned}
u^{\prime \prime}\left(x_{l}, t_{s+1}\right)= & \left(t_{s+1}-t_{s}\right) \sum_{n=1}^{2^{k-1}} \sum_{m=0}^{M-1} c_{n m} \psi_{n m}\left(x_{l}\right)+u^{\prime \prime}\left(x_{l}, t_{s}\right), \\
u^{\prime}\left(x_{l}, t_{s+1}\right)= & \left(t_{s+1}-t_{s}\right) \sum_{n=1}^{2^{k-1}} \sum_{m=0}^{M-1} c_{n m} p_{n m}\left(x_{l}\right)+u^{\prime}\left(x_{l}, t_{s}\right) \\
& -\left(t_{s+1}-t_{s}\right) \sum_{n=1}^{2^{k-1}} \sum_{m=0}^{M-1} c_{n m} q_{n m}(1)+f_{2}\left(t_{s+1}\right) \\
& -f_{2}\left(t_{s}\right)+f_{1}\left(t_{s}\right)-f_{1}\left(t_{s+1}\right),
\end{aligned}
$$




$$
\begin{aligned}
u\left(x_{l}, t_{s+1}\right)= & \left(t_{s+1}-t_{s}\right) \sum_{n=1}^{2^{k-1}} \sum_{m=0}^{M-1} c_{n m} q_{n m}\left(x_{l}\right)+u\left(x_{l}, t_{s}\right) \\
& +f_{1}\left(t_{s+1}\right)-f_{1}\left(t_{s}\right) \\
& +x_{l}\left[-\left(t_{s+1}-t_{s}\right) \sum_{n=1}^{2^{k-1}} \sum_{m=0}^{M-1} c_{n m} q_{n m}(1)\right] \\
& +x_{l}\left[f_{2}\left(t_{s+1}\right)-f_{2}\left(t_{s}\right)+f_{1}\left(t_{s}\right)-f_{1}\left(t_{s+1}\right)\right], \\
\dot{u}\left(x_{l}, t_{s+1}\right)= & \sum_{n=1}^{2^{k-1}} \sum_{m=0}^{M-1} c_{n m} q_{n m}\left(x_{l}\right)+f_{1}^{\prime}\left(t_{s+1}\right) \\
& +x_{l}\left[-\sum_{n=1}^{2^{k-1}} \sum_{m=0}^{M-1} c_{n m} q_{n m}(1)-f_{1}^{\prime}\left(t_{s+1}\right)+f_{2}^{\prime}\left(t_{s+1}\right)\right] .
\end{aligned}
$$

Similarly we obtain

$$
\begin{aligned}
v^{\prime \prime}\left(x_{l}, t_{s+1}\right)= & \left(t_{s+1}-t_{s}\right) \sum_{n=1}^{2^{k-1}} \sum_{m=0}^{M-1} d_{n m} \psi_{n m}\left(x_{l}\right)+v^{\prime \prime}\left(x_{l}, t_{s}\right) \\
v^{\prime}\left(x_{l}, t_{s+1}\right)= & \left(t_{s+1}-t_{s}\right) \sum_{n=1}^{2^{k-1}} \sum_{m=0}^{M-1} d_{n m} p_{n m}\left(x_{l}\right)+v^{\prime}\left(x_{l}, t_{s}\right) \\
& -\left(t_{s+1}-t_{s}\right) \sum_{n=1}^{2^{k-1}} \sum_{m=0}^{M-1} d_{n m} q_{n m}(1) \\
& +g_{2}\left(t_{s+1}\right)-g_{2}\left(t_{s}\right)+g_{1}\left(t_{s}\right)-g_{1}\left(t_{s+1}\right) \\
v\left(x_{l}, t_{s+1}\right)= & \left.t_{s+1}-t_{s}\right) \sum_{n=1}^{2^{k-1}} \sum_{m=0}^{M-1} d_{n m} q_{n m}\left(x_{l}\right)+v\left(x_{l}, t_{s}\right)+g_{1}\left(t_{s+1}\right)-g_{1}\left(t_{s}\right) \\
& +x_{l}\left[-\left(t_{s+1}-t_{s}\right) \sum_{n=1}^{2^{k-1}} \sum_{m=0}^{M-1} d_{n m} q_{n m}(1)\right] \\
& +x_{l}\left[g_{2}\left(t_{s+1}\right)-g_{2}\left(t_{s}\right)+g_{1}\left(t_{s}\right)-g_{1}\left(t_{s+1}\right)\right] \\
& 2^{k-1} \sum_{n-1}^{M-1} d_{n m} q_{n m}\left(x_{l}\right)+g_{1}^{\prime}\left(t_{s+1}\right) \\
& \sum_{n=1} \sum_{m=0} x_{l}\left[-\sum_{n=1}^{2^{k-1}} \sum_{m=0}^{M-1} d_{n m} q_{n m}(1)-g_{1}^{\prime}\left(t_{s+1}\right)+g_{2}^{\prime}\left(t_{s+1}\right)\right]
\end{aligned}
$$

for $v(x, t)$. Based on the Eqs. (3.9)-(3.12) and (3.13)-(3.16) we will use following equations.

$$
\begin{aligned}
& \dot{u}\left(x_{l}, t_{s+1}\right)=u^{\prime \prime}\left(x_{l}, t_{s+1}\right)-\eta u\left(x_{l}, t_{s}\right) u^{\prime}\left(x_{l}, t_{s}\right)-\alpha\left[u\left(x_{l}, t_{s}\right) v\left(x_{l}, t_{s}\right)\right]_{x} \\
& \dot{v}\left(x_{l}, t_{s+1}\right)=v^{\prime \prime}\left(x_{l}, t_{s+1}\right)-\xi v\left(x_{l}, t_{s}\right) v^{\prime}\left(x_{l}, t_{s}\right)-\beta\left[u\left(x_{l}, t_{s}\right) v\left(x_{l}, t_{s}\right)\right]_{x}
\end{aligned}
$$


Now by substituting (3.9)-(3.12) and (3.13)-(3.16) into (3.17) we obtain

$$
\begin{aligned}
\sum_{n=1}^{2^{k-1}} \sum_{m=0}^{M-1} c_{n m}\left[q_{n m}\left(x_{l}\right)-x_{l} q_{n m}(1)-\Delta t \psi_{n m}\left(x_{l}\right)\right] & =u^{\prime \prime}\left(x_{l}, t_{s}\right)-\eta u\left(x_{l}, t_{s}\right) u^{\prime}\left(x_{l}, t_{s}\right) \\
& -\alpha\left[u\left(x_{l}, t_{s}\right) v\left(x_{l}, t_{s}\right)\right]_{x}-f_{1}^{\prime}\left(t_{s+1}\right) \\
& +x_{l}\left[f_{1}^{\prime}\left(t_{s+1}\right)-f_{2}^{\prime}\left(t_{s+1}\right)\right] \\
\sum_{n=1}^{2^{k-1}} \sum_{m=0}^{M-1} d_{n m}\left[q_{n m}\left(x_{l}\right)-x_{l} q_{n m}(1)-\Delta t \psi_{n m}\left(x_{l}\right)\right] & =v^{\prime \prime}\left(x_{l}, t_{s}\right)-\eta v\left(x_{l}, t_{s}\right) v^{\prime}\left(x_{l}, t_{s}\right) \\
& -\alpha\left[u\left(x_{l}, t_{s}\right) v\left(x_{l}, t_{s}\right)\right]_{x}-g_{1}^{\prime}\left(t_{s+1}\right) \\
& +x_{l}\left[g_{1}^{\prime}\left(t_{s+1}\right)-g_{2}^{\prime}\left(t_{s+1}\right)\right]
\end{aligned}
$$

where $\Delta t=t_{s+1}-t_{s}$. From the system (3.18) the wavelet coefficients $c_{n m}$ and $d_{n m}$ can be successively calculated. This computation starts with the following initial values.

$$
\begin{aligned}
u\left(x_{l}, 0\right)=\psi_{1}\left(x_{l}\right), & v\left(x_{l}, 0\right)=\psi_{2}\left(x_{l}\right), \\
u^{\prime}\left(x_{l}, 0\right) & =\psi_{1}^{\prime}\left(x_{l}\right), \quad v^{\prime}\left(x_{l}, 0\right)=\psi_{2}^{\prime}\left(x_{l}\right), \\
u^{\prime \prime}\left(x_{l}, 0\right) & =\psi_{1}^{\prime \prime}\left(x_{l}\right), \quad v^{\prime \prime}\left(x_{l}, 0\right)=\psi_{2}^{\prime \prime}\left(x_{l}\right) .
\end{aligned}
$$

We collocate $x$ at $x_{l}=(l-0.5) / m^{\prime}, l=1,2, \ldots, m^{\prime}$ collocation points where $m^{\prime}=2^{k-1} M$.

\section{Numerical results and discussion}

To show the performance of suggested method as compared with the exact solution we are going to use the norms $L_{2}$ and $L_{\infty}$ defined by

$$
\begin{aligned}
L_{2} & =\sqrt{\frac{\sum_{i=1}^{m^{\prime}}\left|u_{i}^{\text {exact }}-u_{i}^{\text {num }}\right|^{2}}{\sum_{i=1}^{m^{\prime}}\left|u_{i}^{\text {exact }}\right|^{2}}}, \\
L_{\infty} & =\max _{i}\left|u_{i}^{\text {exact }}-u_{i}^{\text {num }}\right| .
\end{aligned}
$$

We have executed our computations on Intel Core i5-2410M 2.3Ghz and 4GB (667Mhz) of RAM with the codes implemented in free software package GNU Octave and Python programming language. Graphical outputs were generated by Matplotlib package [15].

\subsection{Problem 1.}

We firstly consider the coupled Burgers' equation (1.1), (1.2) for $\alpha=\beta=\frac{5}{2}$ and $\eta=\xi=$ $-2,[1]$. So we have

$$
\begin{gathered}
u_{t}-u_{x x}-2 u u_{x}+\frac{5}{2}(u \cdot v)_{x}=0, \\
v_{t}-v_{x x}-2 v v_{x}+\frac{5}{2}(u . v)_{x}=0 .
\end{gathered}
$$

The exact solution of the coupled system for $x \in[0,1]$, is

$$
u(x, t)=v(x, t)=\lambda\left[1-\tanh \left(\frac{3}{2} \lambda(40(x-0.5)-3 \lambda t)\right)\right],
$$

boundary conditions and initial conditions are determined from the exact solution and $\lambda$ is an arbitrary constant. This problem has large gradients moving rightward with constant velocity. In Table 1, we show the obtained results for various values of $\lambda$. It can be seen from the Table 1 that by increasing the number of collocation points one can achieve more accurate results. On the other hand as time grows errors get larger. Measured cpu times 
are also given in Table 1 which are quite small. In Fig. 1, we plot the numerical solution for $\lambda=0.05,0.10 .4,0.8$ and $\Delta t=0.005$ at $t=3$ and $t=5$. We see that for greater values of $\lambda$, large gradient regions occur in the solution. The present method is capable of analyzing the large gradient regions that occur in the solution which is an indicator of the efficiency of a numerical method according to the Vasilyev and Paolucci [43] and Basdevant et al. [5].
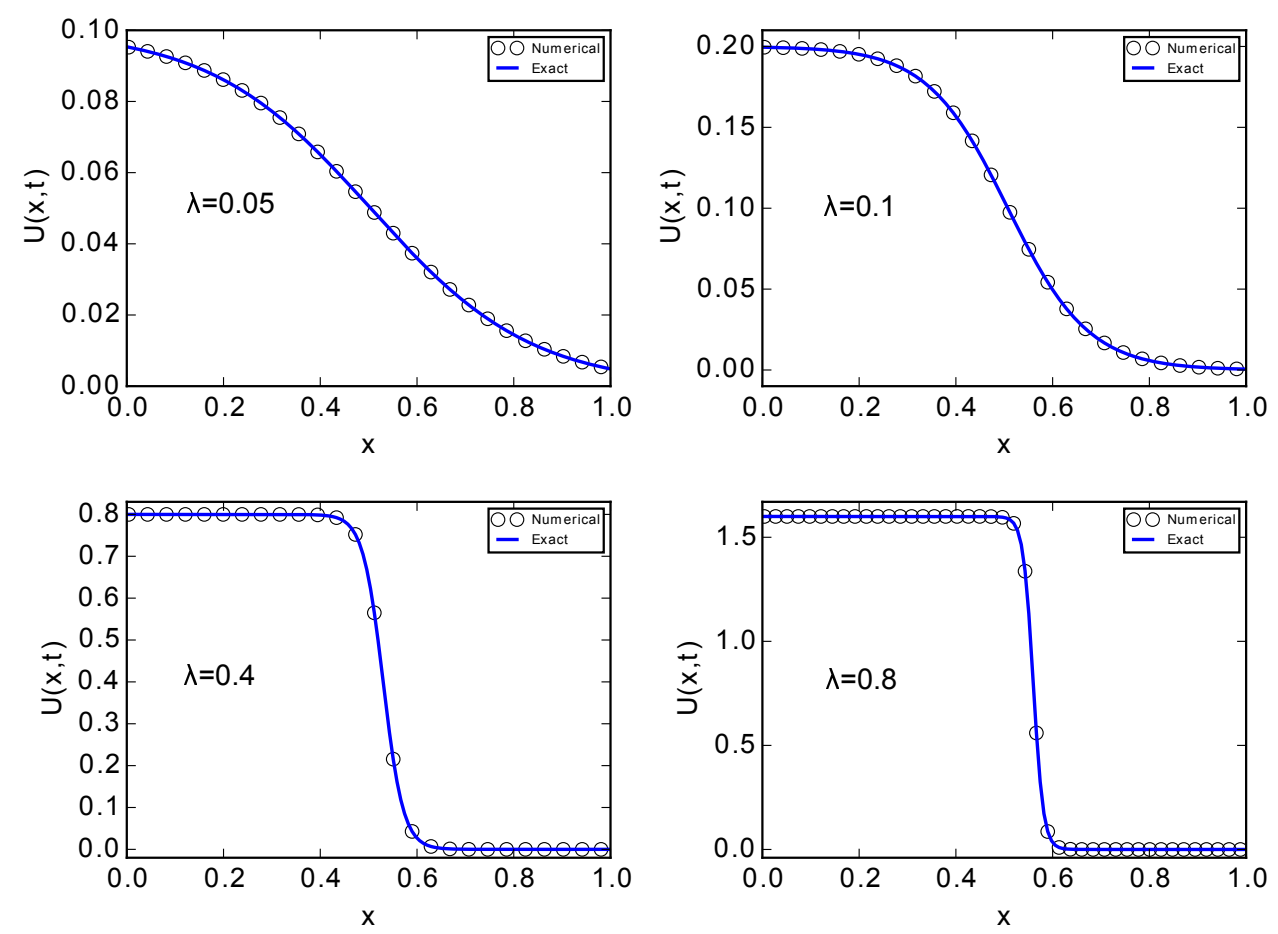

Figure 1. Numerical solution of problem 1 for $\lambda=0.05,0.10 .4,0.8$ and $\Delta t=0.01$ at $t=1$ with $m^{\prime}=128$.

Table 1. Errors for various values of parameters of problem 1.

\begin{tabular}{|c|c|c|c|c|c|c|c|}
\hline & & \multicolumn{2}{|c|}{$k=5, M=4, m^{\prime}=64$} & \multicolumn{2}{|c|}{$k=6, M=4, m^{\prime}=128$} & \multicolumn{2}{|c|}{$k=7, M=4, m^{\prime}=256$} \\
\hline & & $t=3$ & $t=5$ & $t=3$ & $t=5$ & $t=3$ & $t=5$ \\
\hline \multirow{2}{*}{$\lambda=0.1$} & $L_{2}$ & $8.5052 \mathrm{e}-6$ & $1.2956 \mathrm{e}-5$ & $8.4321 \mathrm{e}-6$ & $1.2802 \mathrm{e}-5$ & $8.4272 \mathrm{e}-6$ & $1.2792 \mathrm{e}-5$ \\
\hline & $L_{\infty}$ & $2.7482 \mathrm{e}-6$ & $4.2070 \mathrm{e}-6$ & $2.7140 \mathrm{e}-6$ & $4.1204 \mathrm{e}-6$ & $2.7133 \mathrm{e}-6$ & $4.1136 \mathrm{e}-6$ \\
\hline \multirow{3}{*}{$\lambda=0.8$} & $L_{2}$ & $1.0348 \mathrm{e}-2$ & $1.3720 \mathrm{e}-2$ & $1.1782 \mathrm{e}-3$ & $1.3820 \mathrm{e}-3$ & $7.6982 \mathrm{e}-4$ & $7.0642 \mathrm{e}-4$ \\
\hline & $L_{\infty}$ & $7.7311 \mathrm{e}-2$ & $1.1248 \mathrm{e}-1$ & $9.5027 \mathrm{e}-3$ & $1.2746 \mathrm{e}-2$ & $7.0736 \mathrm{e}-3$ & $7.0315 \mathrm{e}-3$ \\
\hline & Cpu time & 0.458 & 0.796 & 0.963 & 1.505 & 2.633 & 4.081 \\
\hline
\end{tabular}

\subsection{Problem 2.}

We consider the coupled Burgers' equation (1.1), (1.2) for $\eta=\xi=-2$ so that equations (1.1), (1.2) take the following form:

$$
\begin{aligned}
u_{t}-u_{x x}-2 u u_{x}+\alpha(u . v)_{x} & =0, \\
v_{t}-v_{x x}-2 v v_{x}+\beta(u . v)_{x} & =0 .
\end{aligned}
$$


The exact solution of this equation is given in [41]

$$
\begin{aligned}
& u(x, t)=a_{0}(1-\tanh (A(20(x-0.5)-2 A t))) \\
& v(x, t)=a_{0}\left(\left(\frac{2 \beta-1}{2 \alpha-1}\right)-\tanh (A(20(x-0.5)-2 A t))\right)
\end{aligned}
$$

where

$$
a_{0}=0.05, A=\frac{1}{2} a_{0}\left(\frac{4 \alpha \beta-1}{2 \alpha-1}\right)
$$

and $x \in[0,1]$. The initial and boundary conditions are taken from the exact solution. In Table 2, we tabulated and compared the obtained results from the present method with Chebyshev spectral collocation method [21], cubic B-spline collocation method [28], Galerkin quadratic B-spline finite element method [23] and Fourier pseudospectral method [34]. We take $k=4, M=2$ and $\Delta t=0.01$. It can be seen from the Table 2 , the $L_{\infty}$ error norm obtained by the present method is smaller than those obtained by the existing methods and even for the small number of collocation points one can achieve the accuracy of the existing methods in the literature. In Table 3 , we compare the $L_{\infty}$ error norms for $\alpha=0.1, \beta=0.3, k=4, M=2$ and $\Delta t=0.001$ with the ones obtained by Haar wavelet method [22]. The superiority of the present method over Haar wavelet method in the sense of accuracy is clearly seen from Table 3 . The numerical solutions at $t=1$, are shown in Fig. 2 for $k=4, M=2$ and $\Delta t=0.01$. 


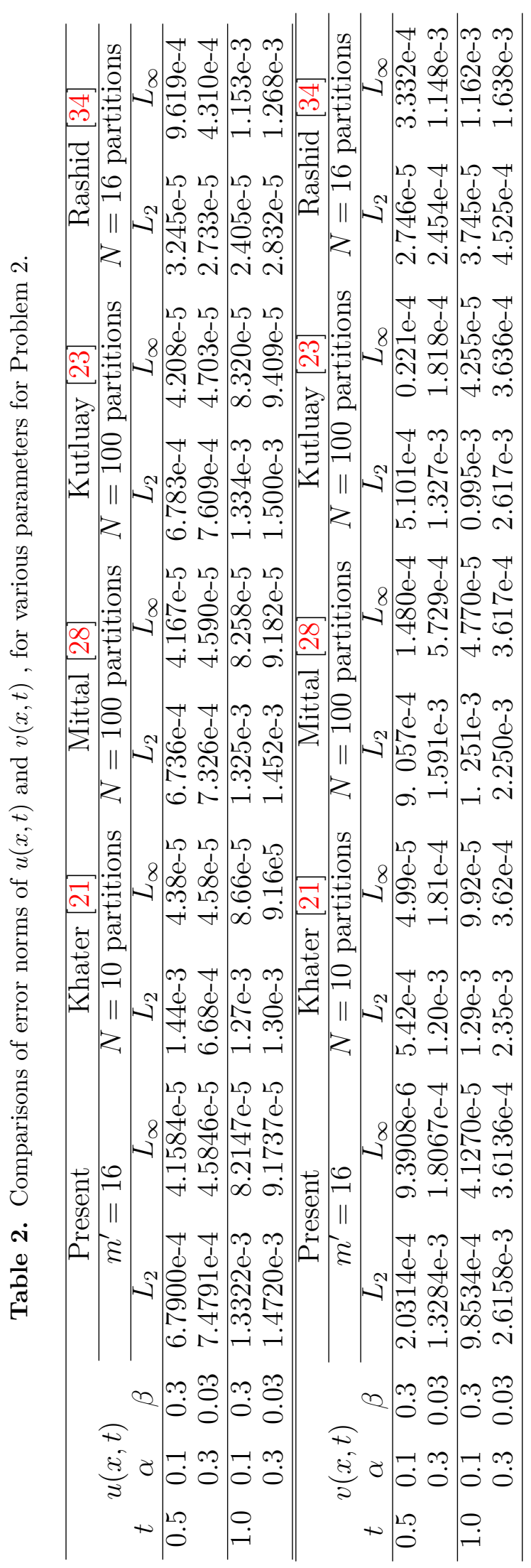


Table 3. Comparison of error norms for $\alpha=0.1, \beta=0.3$ and $\Delta t=0.001$ at various time levels for Problem 2.

\begin{tabular}{|c|c|c|c|c|c|}
\hline \multirow[b]{2}{*}{$t$} & \multicolumn{2}{|c|}{$L_{\infty}(u)$} & \multicolumn{2}{|c|}{$L_{\infty}(v)$} & \multirow{2}{*}{$\begin{array}{l}\text { Cpu times } \\
\text { for the }\end{array}$} \\
\hline & Present & Haar & Present & Haar & \\
\hline & $m^{\prime}=16$ & $N=64$ & $m^{\prime}=16$ & $N=64$ & present method \\
\hline 0.5 & $4.1638 \mathrm{e}-5$ & $5.675 \mathrm{e}-5$ & $2.1915 \mathrm{e}-5$ & $3.679 \mathrm{e}-5$ & 0.2089 \\
\hline 2 & $1.6239 \mathrm{e}-4$ & $2.085 \mathrm{e}-4$ & $7.9455 \mathrm{e}-5$ & $1.359 \mathrm{e}-4$ & 0.8045 \\
\hline 3 & $2.3962 \mathrm{e}-4$ & $3.006 \mathrm{e}-4$ & $1.1427 \mathrm{e}-4$ & $2.049 \mathrm{e}-4$ & 1.2080 \\
\hline
\end{tabular}
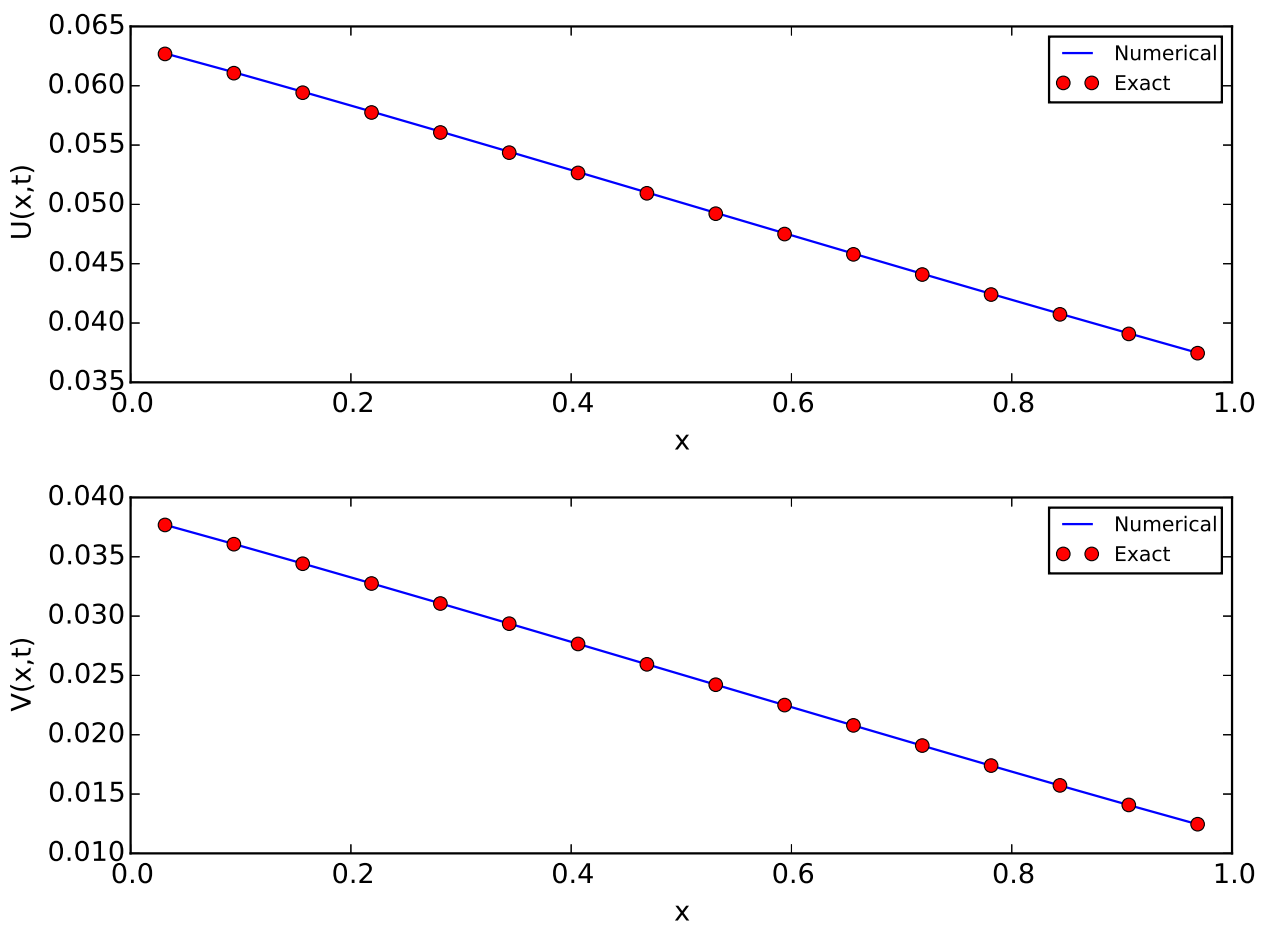

Figure 2. Numerical solutions of problem 2 for $\alpha=0.1, \beta=0.3, \Delta t=0.01$ at $t=1$.

\subsection{Problem 3.}

Lastly we consider the coupled Burgers' equation (1.1), (1.2) for $\alpha=\beta=1$ and $\eta=\xi=-2$ so that equations (1.1), (1.2) take the following form:

$$
\begin{aligned}
u_{t}-u_{x x}-2 u u_{x}+(u . v)_{x} & =0, \\
v_{t}-v_{x x}-2 v v_{x}+(u . v)_{x} & =0 .
\end{aligned}
$$

The exact solution is $u(x, t)=v(x, t)=e^{-t} \sin (2 \pi(x-0.5)), x \in[0,1]$. The initial and boundary conditions are taken from the exact solution. Comparison of the error norms at each time for $k=6, M=2$ and $\Delta t=0.001$ is given in Table 4 . The obtained results by the present method are in good agreement with Haar wavelet method [29] and are better than Finite element method [23]. The physical behavior of numerical solutions for $\alpha=\beta=1$ and $\eta=\xi=-2$ between $t=0$ and $t=2$ and for $\alpha=3, \beta=2$ and $\eta=1, \xi=-2$ between $t=0$ and $t=1.5$ are depicted with contour forms in Fig. 3 and Fig. (4) respectively. 


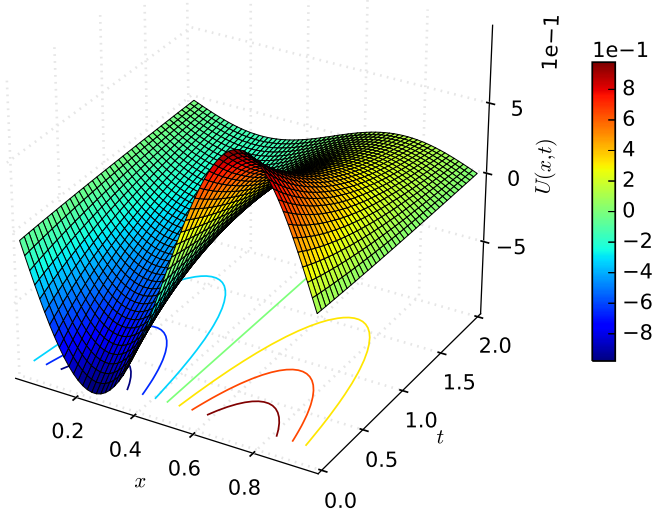

Figure 3. Numerical solution $u(x, t)$ of problem 3 for $\Delta t=0.025, m^{\prime}=64$, $\alpha=\beta=1$ and $\eta=\xi=-2$.

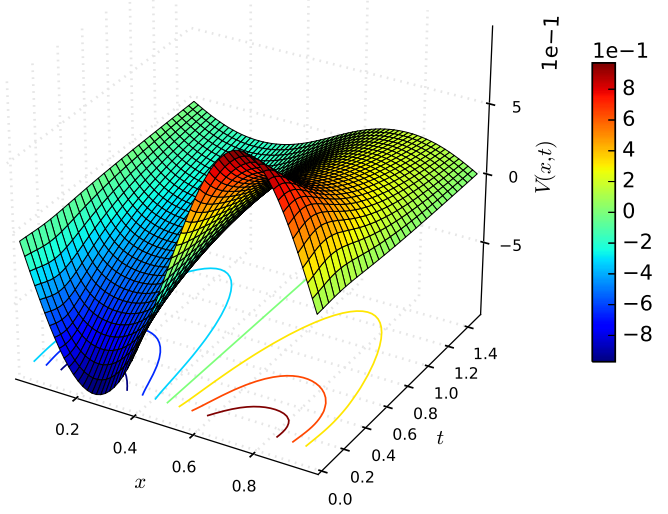

Figure 4. Numerical solution $v(x, t)$ of problem 3 for $\Delta t=0.025, m^{\prime}=64$, $\alpha=3, \beta=2$ and $\eta=1, \xi=-2$.

Table 4. Comparisons of error norms for $\Delta t=0.001$ at different times for problem 3 .

\begin{tabular}{|c|c|c|c|c|c|c|c|}
\hline & Kutlue & [23] & \multirow{2}{*}{\multicolumn{2}{|c|}{$\frac{\text { Mittal }[29]}{\text { (64 partitions) }}$}} & \multirow{2}{*}{\multicolumn{2}{|c|}{$\begin{array}{c}\text { Present method } \\
\text { (64 partitions) } \\
\end{array}$}} & \multirow{3}{*}{$\begin{array}{c}\text { Cpu Time } \\
\text { of Present } \\
\text { Method }\end{array}$} \\
\hline & \multicolumn{2}{|c|}{ (100 partitions) } & & & & & \\
\hline & $L_{2}$ & $L_{\infty}$ & $L_{2}$ & $L_{\infty}$ & $L_{2}$ & $L_{\infty}$ & \\
\hline$t=0.01$ & & & $1.876 \mathrm{e}-4$ & $1.986 \mathrm{e}-5$ & $4.9971 \mathrm{e}-6$ & $5.0040 \mathrm{e}-6$ & 0.048 \\
\hline$t=0.1$ & $1.396 \mathrm{e}-4$ & $3.984 \mathrm{e}-4$ & $1.943 \mathrm{e}-4$ & $1.382 \mathrm{e}-5$ & $5.0122 \mathrm{e}-5$ & $4.9327 \mathrm{e}-5$ & 0.119 \\
\hline$t=0.5$ & $2.473 \mathrm{e}-4$ & $2.869 \mathrm{e}-4$ & $2.232 \mathrm{e}-4$ & $2.119 \mathrm{e}-5$ & $2.1336 \mathrm{e}-4$ & $1.4195 \mathrm{e}-4$ & 0.385 \\
\hline$t=1$ & $3.530 \mathrm{e}-4$ & $1.786 \mathrm{e}-4$ & $2.676 \mathrm{e}-4$ & $1.552 \mathrm{e}-5$ & $2.5548 \mathrm{e}-4$ & $9.8263 \mathrm{e}-5$ & 0.712 \\
\hline
\end{tabular}




\section{Conclusion}

In this paper Chebyshev wavelet method is used to get numerical solutions of one dimensional coupled Burgers' equation. In the solution procedure, the highest derivatives that appeared in the equations are expanded into Chebyshev wavelets and with aid of the integrals of Chebyshev wavelets the considered partial differential equations are converted to algebraic system of equations. The proposed method is tested by three examples and obtained results are compared with the exact solution and with those existed in the literature such as Finite element method, Haar wavelet method and Spectral methods. The comparisons show that the present method is quite satisfactory and competitive with other methods. We can give the highlights of the present method as follows:

- The present method can handle boundary conditions easily.

- Computer simulations show that the proposed method is computationally cheap, fast and gives accurate results even in the case of a small number of collocation points.

- The computer implementation of the proposed method is simple and straightforward.

- The present method can also be used for similar partial differential equations from different branches of science and engineering with suitable modifications.

\section{References}

[1] R. Abazari and A. Borhanifar, Numerical study of the solution of the Burgers and coupled Burgers equations by a differential transformation method, Comput. Math. Appl. 59, 2711-2722, 2010.

[2] M.A. Abdou and A.A. Soliman, Variational iteration method for solving Burger's and coupled Burger's equations, J. Comput. Appl. Math. 181, 245-251, 2005.

[3] H. Adibi and P. Assari Chebyshev wavelet method for numerical solution of Fredholm integral equations of the first kind. Math. Prob. Eng. 2010, 1-17, 2010, doi:10.1155/2010/138408.

[4] E. Babolian and F. Fattahzadeh Numerical solution of differential equations by using Chebyshev wavelet operational matrix of integration, Appl. Math. Comput. 188, 417426, 2007.

[5] C. Basdevant, M. Deville, P. Haldenwang and J. M. Lacroix Spectral and finite difference solutions of the Burgers' equation, Comput. Fluids 14, 23-41, 1986.

[6] F. Bulut, Ö. Oruç and A. Esen, Numerical Solutions of Fractional System of Partial Differential Equations By Haar Wavelets, Comput. Model. Eng. Sci. 108 (4), 263-284, 2015.

[7] I. Celik, Haar wavelet approximation for magnetohydrodynamic flow equations, Appl. Math. Model. 37, 3894-3902, 2013.

[8] I. Celik, Chebyshev Wavelet collocation method for solving generalized Burgers-Huxley equation, Math. Methods. Appl. Sci. 39 (3), 366-377, 2015.

[9] C. Chen and C.H. Hsiao, Haar wavelet method for solving lumped and distributed parameter systems, IEE Proc., Control Theory Appl. 144, 87-94, 1997.

[10] I. Daubechies, Ten Lectures on Wavelet, SIAM, Philadelphia, 1992.

[11] M. Dehghan, A. Hamidi and M. Shakourifar, The solution of coupled Burgers' equations using Adomian-Pade technique, Appl. Math. Comput. 189, 1034-1047, 2007.

[12] S.E. Esipov, Coupled Burgers' equations: a model of polydispersive sedimentation, Phys. Rev. Lett. 52, 3711-3718, 1995.

[13] A.K. Gupta and S. Saha Ray, Travelling wave solution of fractional KdV-BurgerKuramoto equation describing nonlinear physical phenomena, AIP Adv. 4, 2014, doi: 10.1063/1.4895910.097120-1-11. 
[14] M.H. Heydari, M.R. Hooshmandasl and F.M. Maalek Ghaini, A new approach of the Chebyshev wavelets method for partial differential equations with boundary conditions of the telegraph type, Appl. Math. Model. 38, 1597-1606, 2014.

[15] J. D. Hunter, Matplotlib: A 2D graphics environment, Comput. Sci. Eng. 9 (3), 90-95, 2007.

[16] I.E. Inan, D. Kaya and Y. Ugurlu, Auto-Bäcklund transformation and similarity reductions for coupled Burger's equation, Appl. Math. Comput. 216, 2507-2511, 2010.

[17] S. Islam, S. Haq and M. Uddin, A mesh free interpolation method for the numerical solution of the coupled nonlinear partial differential equations, Eng. Anal. Bound. Elem. 33, 399-409, 2009.

[18] R. Jiwari, A Haar wavelet quasilinearization approach for numerical simulation of Burgers' equation, Comput. Phys. Commun. 183, 2413-2423, 2012.

[19] D. Kaya, An explicit solution of coupled viscous Burgers' equation by the decomposition method. Int. J. Math. Math. Sci. 27 (11), 675-680, 2001.

[20] A. Kelleci and A. Yildırım, An efficient numerical method for solving coupled Burgers' equation by combining homotopy perturbation and pade techniques, Numer. Methods Partial Differ. Equ. 27 (4), 982-995, 2011.

[21] A.H. Khater, R.S. Temsah and M.M. Hassan, A Chebyshev spectral collocation method for solving Burgers'-type equations, J. Comput. Appl. Math. 222 (2), 333-350, 2008.

[22] M. Kumar and S. Pandit, A composite numerical scheme for the numerical simulation of coupled Burgers' equation, Comput. Phys. Commun. 185 (3), 809-817, 2014.

[23] S. Kutluay and Y. Ucar, Numerical solutions of the coupled Burgers' equation by the Galerkin quadratic B-spline finite element method, Math. Methods Appl. Sci. 36 (17) 2403-2415, 2013.

[24] U. Lepik, Numerical solution of differential equations using Haar wavelets, Math. Comput. Simul. 68, 127-143, 2005.

[25] U. Lepik, Application of the Haar wavelet transform to solving integral and differential Equations, Proc. Estonian Acad. Sci. Phys. Math. 56 (1), 28-46, 2007.

[26] U. Lepik, Numerical solution of evolution equations by the Haar wavelet method, Appl. Math. Comput. 185, 695-704, 2007.

[27] U. Lepik, Solving PDEs with the aid of two-dimensional Haar wavelets, Comput. Math. Appl. 61, 1873-1879, 2011.

[28] R.C. Mittal and G. Arora, Numerical solution of the coupled viscous Burgers' equation, Commun. Nonlinear Sci. Numer. Simul. 16, 1304-1313, 2011.

[29] R.C. Mittal, H. Kaur, and V. Mishra, Haar wavelet-based numerical investigation of coupled viscous Burgers' equation, Int. J. Comput. Math. 92 (8), 1643-1659, 2015, doi: 10.1080/00207160.2014.957688

[30] J. Nee and J. Duan, Limit set of trajectories of the coupled viscous Burgers' equations, Appl. Math. Lett. 11 (1), 57-61, 1998.

[31] Ö. Oruç, F. Bulut and A. Esen, A haar wavelet-finite difference hybrid method for the numerical solution of the modified Burgers' equation, J. Math. Chem. 53 (7), 1592-1607, 2015.

[32] Ö. Oruç, F. Bulut and A. Esen, Numerical Solutions of Regularized Long Wave Equation By Haar Wavelet Method, Mediterr. J. Math. 13 (5), 3235-3253, 2016.

[33] A. Rashid, M. Abbas, A.I.Md. Ismail and A. Abd Majid, Numerical solution of the coupled viscous Burgers equations by Chebyshev-Legendre Pseudo-Spectral method, Appl. Math. Comput. 245, 372-381, 2014.

[34] A. Rashid and A.I.B.M. Ismail, A Fourier pseudospectral method for solving coupled viscous Burgers equations, Comput. Methods Appl. Math. 9 (4), 412-420, 2009.

[35] M. Razzaghi and S. Yousefi, Legendre wavelets direct method for variational problems, Math. Comput. Simul. 53, 185-192, 2000. 
[36] M. Razzaghi and S. Yousefi, Legendre wavelets operational matrix of integration, Int. J. Syst. Sci. 32 (4), 49-502, 2001.

[37] Z. Rong-Pei, Y. Xi-Jun and Z. Guo-Zhong, Local discontinuous Galerki nmethod for solving Burgers and coupled Burgers equations, Chin. Phys. B. 20 (11), 110205-1-6, 2011.

[38] P.K. Sahu and S. Saha Ray, Legendre wavelets operational method for the numerical solutions of nonlinear Volterra integro-differential equations system, Appl. Math. Comput. 256, 715-723, 2015.

[39] P.K. Sahu and S. Saha Ray, Two dimensional Legendre wavelet method for the numerical solutions of fuzzy integro-differential equations, J. Intell. Fuzzy Syst. 28, 12711279, 2015.

[40] Z. Shi, Y. Cao, and Q.J. Chen, Solving 2D and 3D Poisson equations and biharmonic equations by the Haar wavelet method, Appl. Math. Model. 36, 5143-5161, 2012.

[41] A.A. Soliman, The modified extended tanh-function method for solving Burgers-type equations, Physica A 361, 394-404, 2006.

[42] V.K. Srivastava, M.K. Awasthi and M. Tamsir, A fully implicit Finite-difference solution to one dimensional Coupled Nonlinear Burgers' equations, Int. J. Math. Sci. 7 (4) 2013.

[43] O.V. Vasilyev and S. Paolucci, A Dynamically Adaptive Multilevel Wavelet Collocation Method for Solving Partial Differential Equations in a Finite Domain, J. Comput. Phys. 125, 498-512, 1996.

[44] Y. Wang and Q. Fan, The second kind Chebyshev wavelet method for solving fractional differential equations, Appl. Math. Comput. 218, 8592-8601, 2012.

[45] C. Yang and J. Hou, Chebyshev wavelets method for solving Bratu's problem, Bound. Value. Probl. 142, 1-9, 2013.

[46] F. Zhou and X. Xu, Numerical solution of the convection diffusion equations by the second kind Chebyshev wavelets, Appl. Math. Comput. 247, 353-367, 2014.

[47] L. Zhu and Q. Fan, Solving fractional nonlinear Fredholm integro-differential equations by the second kind Chebyshev wavelet, Commun. Nonlinear Sci Numer. Simul. 17, 2333-2341, 2012. 